\title{
Técnica Tied Out Open Sky: fixação iriana de lente intraocular combinada com transplante penetrante de córnea
}

\author{
Tied Out Open Sky technique: new method for iris fixation \\ of intraocular lens in the absence of capsular support \\ during penetrating keratoplasty
}

Victor Andriguetti Coronado Antunes', Wilson Takashi Hida², Danilo da Costa Sousa ${ }^{3}$, Iris Yamane ${ }^{4}$, Fernando Moro ${ }^{1}$, Karine Borges Marques Moysés ${ }^{1}$, Tadeu Cvintal ${ }^{1}$, Luciana Malta de Alencar ${ }^{5}$, Celso Takashi Nakano ${ }^{4}$, Patrick Frensel Tzelikis ${ }^{7}$

\section{Resumo}

O objetivo é descrever uma nova técnica cirúrgica para fixação iriana de lente intraocular durante transplante de córnea em pacientes sem suporte capsular. A técnica de fixação iriana Tied Out Open Sky é uma técnica de fácil execução comparado com as técnicas já descritas, dispensando manobras cirúrgicas complexas, minimizando a manipulação e as complicações pós-operatórias.

Descritores: Ceratoplastia penetrante; Transplante de córnea; Subluxação do cristalino; Íris/cirurgia; Afacia; Ferimentos e lesões; Relatos de casos

\section{Abstract}

To describe a new method for iris fixation of intraocular lens in the absence of capsular support during penetrating keratoplasty. Its a new technique of iris fixation of intraocular lens without capsular support during penetrating keratoplasty. This technique is used in cases with a healthy iris and partial or total absence of capsular support during penetrating keratoplasty.Tied Out Open Sky is a technique easy to perform for iris fixation of intraocular lens during penetrating keratoplasty. The big advantage is being able to tie off the intraocular lens off the eye and fasten it securely.

Keywords: Keratoplasty, penetrating; Corneal transplantation; Lens subluxation; Aphakia; Iris/surgery; Case reports

\footnotetext{
1'Assistente do Departamento de Córnea do Instituto de Oftalmologia Tadeu Cvintal - São Paulo (SP), Brasil;

${ }^{2}$ Pos-graduando (Doutorado) do Setor da Catarata da Faculdade de Medicina da Universidade de São Paulo (USP) - São Paulo (SP),

Brasil; Chefe do Setor de Catarata do Hospital Oftalmologico de Brasília (HOB) - (DF), Brasil;

${ }^{3}$ Estagiário do Departamento de Catarata e Refrativa do Hospital Oftalmológico de Brasília (DF), Brasil;

${ }^{4}$ Estagiário do Setor da Catarata da Faculdade de Medicina da Universidade de São Paulo (USP) - São Paulo (SP), Brasil;

${ }^{5}$ Doutora do Setor de Glaucoma da Faculdade de Medicina da Universidade de São Paulo (USP) - São Paulo (SP),Brasil; Chefe do

Setor de Glaucoma do Hospital Oftalmologico de Brasilia (HOB) - (DF), Brasil;

${ }^{6}$ Assistente do Departamento de Córnea e Catarata do Hospital Oftalmológico de Brasília (DF),Brasil.
}

Os autores declaram inexistir conflitos de interesses

Recebido para publicação em: 2/8/2011 - Aceito para publicação em 3/10/2011 


\section{INTRODUÇÃO}

$\mathbf{O}$ implante de lente intraocular em pacientes sem suporte capsular adequado é usualmente complexo, pois estes olhos geralmente possuem patologias pré-existentes e a técnica cirúrgica de implante secundário de lentes intraoculares pode estar prejudicada por estas patologias ${ }^{(1)}$. A eficiência da ceratoplastia penetrante combinada com o implante ou a troca de lentes intraoculares em casos de ceratopatia bolhosa já foi amplamente demonstrado ${ }^{(2-8)}$.

Teoricamente, o implante de lentes de câmara posterior causam menores danos a córnea, íris e estruturas do ângulo, reduzindo as chances de descompensação da córnea, inflamação e elevação da pressão ${ }^{(9,10)}$.

A escolha da técnica adequada para a fixação de lente durante a ceratoplastia penetrante vai depender de alguns fatores. Em comparação com as lentes de câmara anterior, as lentes de câmara posterior localizamse mais próximas ao ponto nodal, aumentando as chances de reabilitação visual. Outra vantagem destas lentes é o anteparo produzido pela própria íris entre a lente e o endotélio, simulando a anatomia de um olho saudável e reduzindo a agressão às estruturas anatômicas do segmento anterior.

A técnica de fixação escleral, utilizada em maior escala por não depender da integridade iriana, possui maior risco de hemorragias intraoculares e descolamento de retina ${ }^{(11-13)}$. As principais complicações dessa técnica são decorrentes da transfixação da esclera, com lesão vascular, rotura e descolamento de retina e criação de um pertuito para dentro do olho.

Uma outra alternativa é a fixação iriana de lente intraocular. Apesar dos riscos de atrofia iriana e irite crônica, estudos mostraram que não há aumento da incidência de edema macular cistóide e descompensação corneana com esta técnica ${ }^{(14)}$.

Através deste relato de caso, pretendemos demonstrar uma variação da técnica cirúrgica de fixação iriana em transplante penetrante de córnea que poderá facilitar a técnica, devido à amarra ser realizada fora do olho e, por isso assim chamada de Tied Out Open Sky .

\section{Relato de caso}

Paciente com 41 anos, branca, sexo feminino, solteira, aposentada, natural e procedente de São Paulo, diabética há 7 anos, com história de trauma perfurante em olho esquerdo na infância aos 10 anos de idade. Na época realizou cirurgia para reparar o trauma, evoluindo com baixa acuidade visual severa. Deu entrada em nosso serviço com queixa de baixa acuidade visual e sensação de corpo estranho no olho esquerdo. Ao exame, apresentava acuidade visual de 20/20 no olho direito e movimento de mãos no olho esquerdo. Apresentava uma exotropia do olho esquerdo de 30 dioptrias prismáticas. Tonometria de aplanação de $13 \mathrm{mmHg}$ em ambos os olhos. Reflexo pupilar em olho direito normal e ausente em olho esquerdo. Biomicroscopia apresentava em olho esquerdo, ceratopatia bolhosa com microbolhas epiteliais difusas, discreta vascularização corneana às 5 horas, iridectomia setorial nasal e cristalino em reabsorção. (Figura 1) Fundoscopia de olho esquerdo apresentando bom reflexo vermelho sendo impossível vizualizar detalhes. Ecografia ocular apresentou cristalino em reabsorção, opacidades vítreas móveis, retina aplicada e nervo óptico normal.

Apesar da possibilidade de ambliopia, optamos pela tentativa de reabilitação visual com transplante penetrante de córnea e implante secundário de lente intraocular.

\section{O procedimento cirúrgico}

Foi realizado sutura de anel de Flieringa com fio Mersilene 6.0, a córnea doadora trepanada com lâmina $8.75 \mathrm{~mm}$ e a receptora $8.25 \mathrm{~mm}$. Ao trepanar a córnea receptora, nos deparamos com um cristalino em reabsorção com fragilidade zonular $360^{\circ}$, nos restando a opção de fazer a extração intracapsular do cristalino opaco em reabsorção (Figura 2)

Após a trepanação da córnea receptora, colocamos um gancho do tipo Lester debaixo da íris e com uma leve protrusão produzimos saliência linear que pela visualização, facilita a passagem da agulha com fio de prolene 10-0 (Ethicon 788 Tapercut CIF-4) pela íris e atrás do gancho (Figura 3 ).

O gancho é gentilmente removido, trazendo o fio de prolene na forma de alça (Figura 4). O mesmo procedimento é realizado a $90^{\circ}$ (Figuras 5A e 5B).

Em seguida, a alça de prolene é atada ao háptico da lente intraocular, através de uma laçada. O fio é passado através do orificio localizado no háptico da lente intraocular, específica para este propósito (PC Scleral fixation IOL, UV PMMA, Openflex “C”". Produzida pela Mediphacos, modelo SF-70H, com 13.5 mm de diâmetro e zona óptica de $7.00 \mathrm{~mm}$ ) e esta laçada é passada pela extremidade da próprio háptico, fixando o fio sem a necessidade de anodar. $\mathrm{O}$ mesmo procedimento é realizado com a outro háptico (Figuras 6A, 6B, 6C, 6D).

Assim que os fios foram passados e fixados, a lente foi posicionada posteriormente (Figura 7A) e as extremidades dos fios foram amarradas (Figuras $8 \mathrm{~A} \mathrm{e} 8 \mathrm{~B}$ ). 


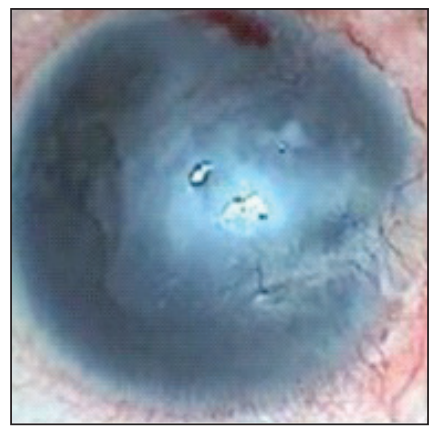

Figura 1: Aspecto inicial ceratopatia bolhosa

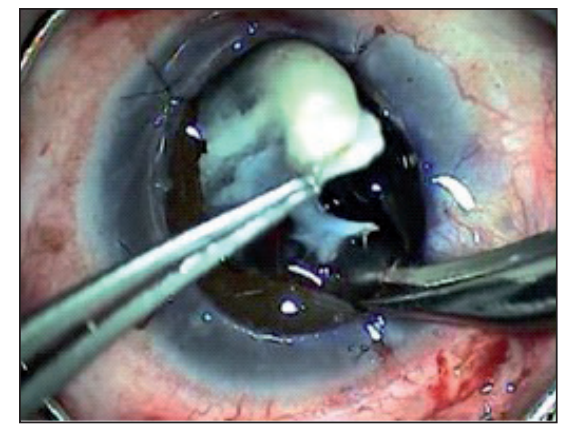

Figura 2: Cristalino em reabsorção

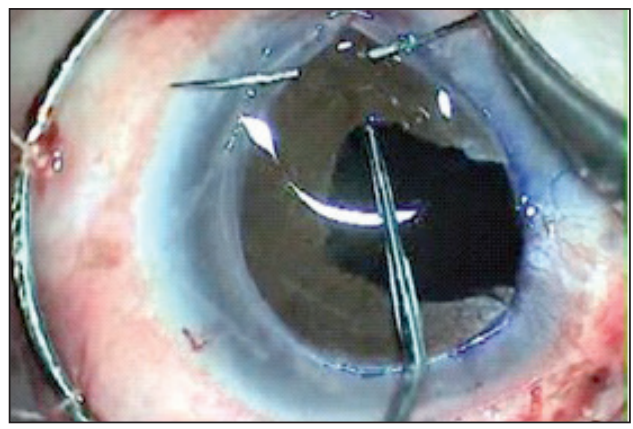

Figura 3: Lester sob a iris, agulha de prolene 10.0 transfixando a iris sob o instrumento

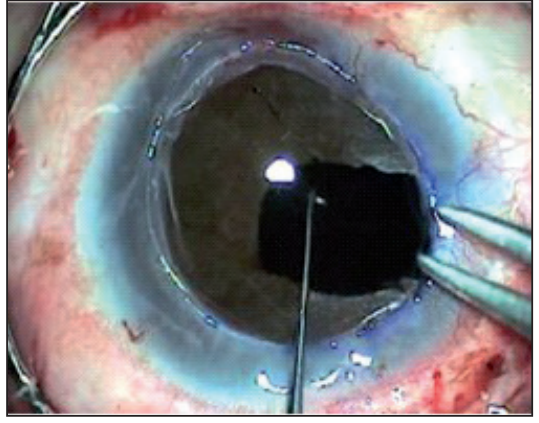

Figura 4: Lester tracionado fio e formando uma alça

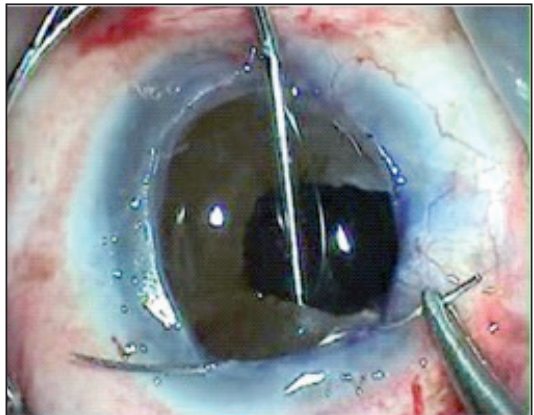

Figura 5-A: Lester sob a iris e transfixação do fio a $90^{\circ}$ do procedimento inicial

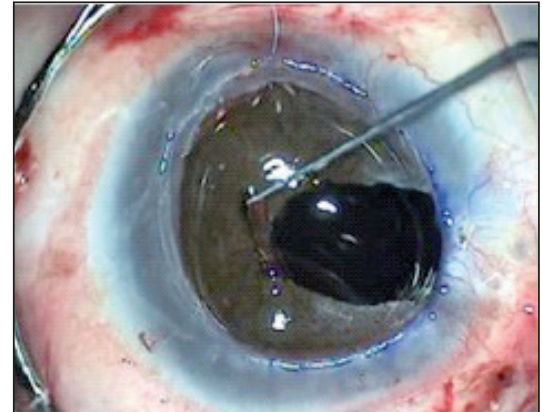

Figura 5-B: Fio sendo trancionado formando uma alça a $90^{\circ}$ do procedimento inicial
Com a lente fixa e estável, realizamos uma iridoplastia, com 2 pontos e o mesmo fio usado na fixação da lente, a fim de restabelecer a pupila (Figuras 9A, 9B). Em seguida, o tranplante de córnea transcorreu normalmente, sendo a córnea doadora suturada com 24 pontos isolados com o fio nylon 10.0.

\section{Discussão}

Worst foi o pioneiro em usar a técnica de fixação de lente intraocular na íris ${ }^{(15)}$. A técnica mais utilizada de fixação iriana de lentes intraoculares sem suporte capsular simultânea à ceratoplastia penetrante é a técnica descrita por Drews em 1987, em que as hápticas da LIO são capturadas na íris e suturadas, estabilizando a lente e permitindo a postergação da óptica com segurança ${ }^{(16)}$.

Acreditamos que a técnica descrita é uma técnica mais segura que a Drews ${ }^{(16)}$, a mais utilizada, pois a fixação da lente intraocular é feita fora do olho, além de não necessitar da integridade do esfíncter pupilar.

A posição ideal para a passagem da sutura de prolene na fixação iriana é a mais periférica que a abertura trepanada da córnea permitir. Nesse ponto a íris tem menos mobilidade, permitindo, em muitos casos, que se dilate a pupila quando necessário e diminuindo a possibilidade de irite causada pela sutura na parte móvel da íris.

A orientação da lente intraocular atrás da íris dependerá das condições anatômicas do segmento anterior. Damos preferência à orientação 6-12 horas, devido à menor possibilidade de inclinação da lente intraocular induzida pela gravidade, mas há descrição de técnicas com fixação na posição 3-9 horas ou oblíqua. Esta decisão também irá depender das condições que a íris se encontra.

Outra técnica também descrita e com certa semelhança a esta exige uma lente biconvexa, três peças de $7 \mathrm{~mm}$ de diâmetro com quatro orifícios de fixação em sua óptica. É usado um fio de prolene 10-0, sendo a sutura passada através da periferia iriana média; primeiramente ela atravessa um dos orifícios da LIO, no sentido ântero-posterior, e na volta atravessa o outro orifício, no sentido póstero-anterior, transfixando novamente. Finalmente, o nó é realizado e o procedimento deve ser repetido no lado oposto ${ }^{(17)}$. Procedimento semelhante é descrito, porém com lentes de apenas dois orifícios para fixação ${ }^{(18)}$.

Acreditamos que a técnica de fixação iriana é de grande utilidade principalmente em casos de ruptura de 


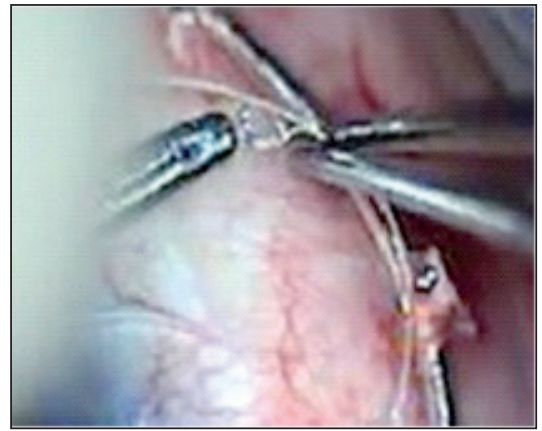

Figura 6-A: Alça do fio passando por orificio da háptica da LIO de fixação

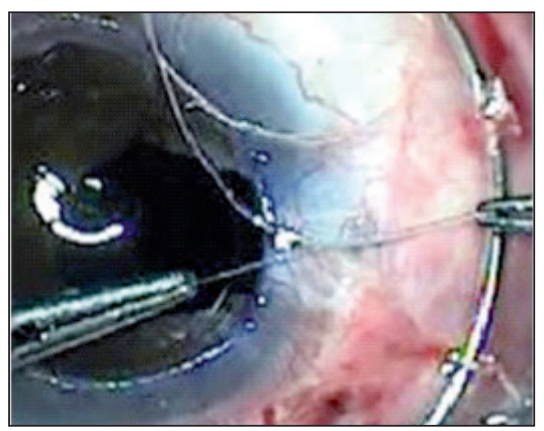

Figura 6-D: O mesmo procedimento é repitido na outra háptica

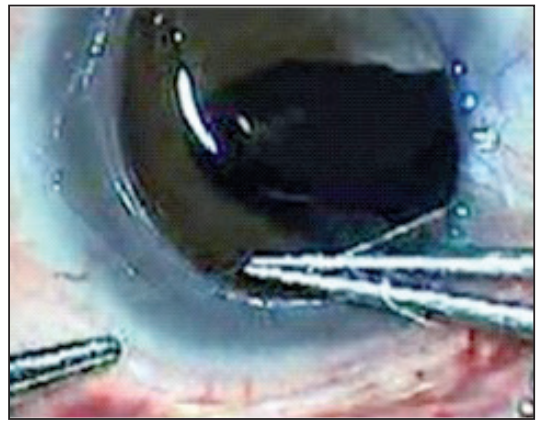

Figura 8-B: Amarra da háptica superior

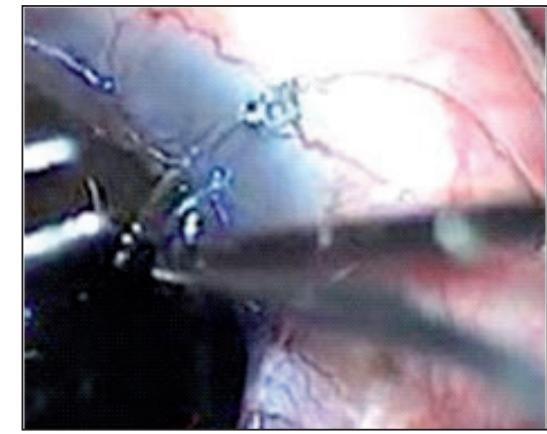

Figura 6-B: Alça sendo levada a extremidade da háptica

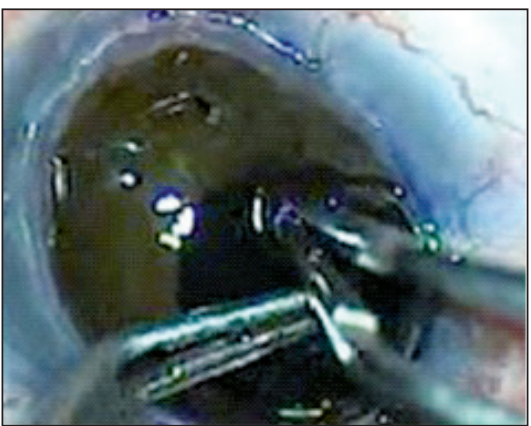

Figura 7: Posteriorização da LIO

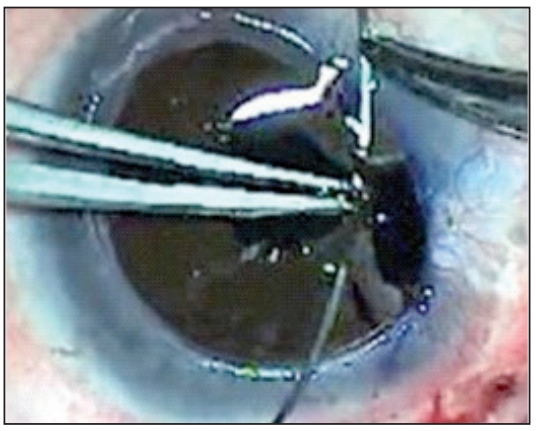

Figura 9-A: Iridoplastia com prolene 10.0

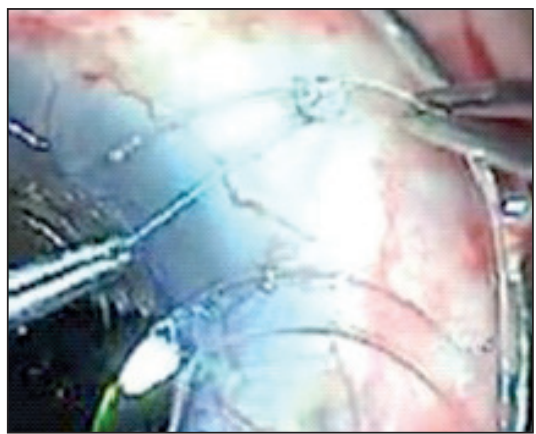

Figura 6-C: Conjunto de fios tracionados travando a alça na háptica

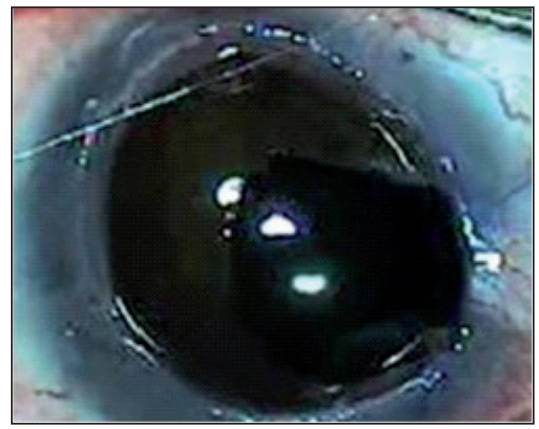

Figura 8-A: Amarra da háptica inferior

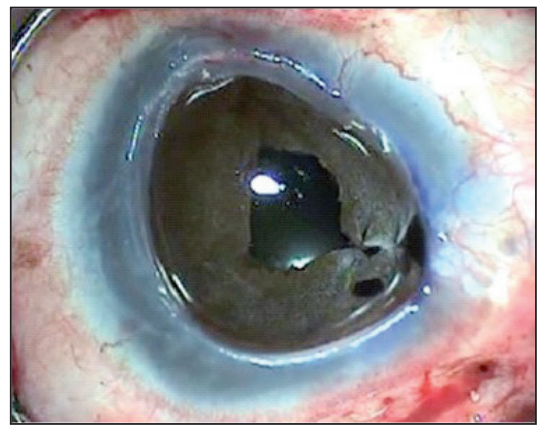

Figura 9-B: Aspecto final da iridoplastia cápsula posterior inadvertida em cirurgia tríplice com comprometimento de suporte capsular e, conseqüentemente, sem que os leitos de fixação tenham sido confeccionados. Outra boa indicação são os casos em que há apenas apoio capsular setorial, com a extremidade oposta sem suporte, podendo ser suturada ao háptico da lente intraocular na íris nessa região, estabilizando o conjunto.

Essa técnica requer alguns critérios para sua realização como afacia sem apoio capsular,íris sadia e com periferia íntegra em no mínimo $270^{\circ}$. A estabilidade da lente intraocular vai depender da integridade do estroma iriano, íris com periferia íntegra em no mínimo $270^{\circ}$. A fixação iriana oferece um risco menor quando atuamos em olhos com risco de descolamento de retina ou que, sabidamente, são portadores de alterações patológicas envolvendo a retina periférica, a ora serrata e a pars plana.

A técnica é chamada de Tied Out Open Sky, pois a fixação da lente intraocular é realizada fora do olho e pode por isso ser usada em casos de transplante penetrante e afacia. Inicialmente usamos um instrumento Lester colocado sob a íris na periferia e o fio é passado. A lente é então atada através da alça do fio que foi retirada da porção inferior da iris. A lente é posteriorizada e a lente é amarrada a íris com as extremidades do fio. 


\section{CONCLUSÃO}

Acreditamos que a técnica Tied Out Open Skyé uma técnica de fixação iriana em transplante de córnea com diversas vantagens em relação às técnicas já descritas. É uma técnica de fácil execução, dispensando manobras cirúrgicas complexas, minimizando a manipulação e as complicações pós-operatórias.

A grande vantagem é poder amarrar a lente fora do olho e fixá-la com segurança,

Necessitamos de um seguimento maior para analisarmos a estabilidade da lente intraocular a longo prazo, além de um número maior de pacientes para analisarmos estatisticamente as possíveis complicações e resultados com esta técnica.

\section{ReferêNCIAS}

1. Zeh WG, Price FW Jr. Iris fixation of posterior chamber intraocular lenses. J Cataract Refract Surg. 2000;26(7):1028-34.

2. Waring GO 3rd, Stulting RD, Street D. Penetrating keratoplasty for pseudophakic corneal edema with exchange of intraocular lenses. Arch Ophthalmol. 1987;105(1):58-62.

3. van der Schaft TL, van Rij G, Renardel de Lavalette JG, Beekhuis WH. Results of penetrating keratoplasty for pseudophakic bullous keratopathy with the exchange of an intraocular lens. Br J Ophthalmol. 1989;73(9):704-8.

4. Binder PS. Secondary intraocular lens implantation during or after corneal transplantation. Am J Ophthalmol. 1985;99(5):515-20.

5. Wong SK, Stark WJ, Gottsch JD, Bernitsky DA, McCartney DL. Use of posterior chamber lenses in pseudophakic bullous keratopathy. Arch Ophthalmol. 1987;105(6):856-8.

6. Lindquist TD, Agapitos PJ, Lindstrom RL, Lane SS, Spigelman AV. Transscleral fixation of posterior chamber intraocular lenses in the absence of capsular support. Ophthalmic Surg. 1989;20(11):769-75. Comment inOphthalmic Surg. 1990;21(5):376.
7. Smiddy WE, Sawusch MR, O'Brien TP, Scott DR, Huang SS. Implantation of scleral-fixated posterior chamber intraocular lenses. J Cataract Refract Surg. 1990;16(6):691-6. Comment inJ Cataract Refract Surg. 1991;17(2):242-3.

8. 'Waring GO 3rd. Management of pseudophakic corneal edema with reconstruction of the anterior ocular segment. Arch Ophthalmol. 1987;105(5):709-15.

9. Apple DJ, Price FW, Gwin T, Imkamp E, Daun M, Casanova R, et al. Sutured retropupillary posterior chamber intraocular lenses for exchange or secondary implantation. The 12th annual Binkhorst lecture, 1988. Ophthalmology. 1989;96(8):1241-7.

10. Solomon K, Gusser JR, Gussler C, Van Meter WS.Incidence and management of complications of transsclerally sutured posterior chamber lenses. J Cataract Refract Surg. 1993;19(4):488-93.

11. Price FW Jr, Whitson WE.Suprachoroidal hemorrhage after placement of a scleral-fixated lens. J Cataract Refract Surg. 1990;16(4):514-5.

12. Lee JG, Lee JH, Chung H. Factors contributing to retinal detachment after transscleral fixation of posterior chamber intraocular lenses. J Cataract Refract Surg. 1998;24(5):697-702.

13. Scott IU, Flynn HW Jr, Feuer W. Endophthalmitis after secondary intraocular lens implantation. A case-report study. Ophthalmology. 1995;102(12):1925-31.

14. Por YM, Lavin MJ.Techniques of intraocular lens suspension in the absence of capsular/zonular support. Surv Ophthalmol. 2005;50(5):429-62. Review.

15. Worst JG. Iris sutures for artificial lens fixation-perlon vs stainless steel. Trans Sect Ophthalmol Am Acad Ophthalmol Otolaryngol. 1976;81(1):OP102-4.

16. Drews RC. Posterior chamber lens implantation during keratoplasty without posterior lens capsule support. Cornea. 1987;6(1):38-40.

17. Davis RM, Best D, Gilbert GE. Comparison of intraocular lens fixation techniques performed during penetrating keratoplasty. Am J Ophthalmol. 1991;111(6):743-9.

18. Olson RJ, Mattingly TP, Waltman SR, Kaufman HE. Aphakic keratoplasty: visual acuity and optical errors. Ophthalmology. 1980;87(7):680-6.

\section{Endereço para correspondência \\ Wilson Takashi Hida}

Rua Afonso de Freitas, 488 - apto 61 - Paraíso

CEP 04006 - 052 - São Paulo (SP), Brasil

E-mail: witaks@yahoo.com.br 